\title{
The Efficacy of Patient Centered Outcomes in Determining the Effectiveness of Surgical Mentorship in Rural Saskatchewan
}

\author{
Pillay $\mathbf{Y}^{1 *}$ and Abeygunawardena $\mathrm{K}^{2}$ \\ ${ }^{1}$ Department of General Surgery, University of \\ Saskatchewan, Canada \\ ${ }^{2}$ University of Toronto, Canada
}

*Correspondling author: Pillay Y, Department of General Surgery, University of Saskatchewan, Prince Albert, SK, Canada

Received: August 23, 2018; Accepted: September 24, 2018; Published: October 01, 2018

\begin{abstract}
Background: Advanced laparoscopic skills are difficult to obtain for practicing surgeons. This issue is exponentially more difficult for surgeons in rural practice. Surgical mentorship is one way in which this can be achieved.

Material and Methods: A surgical mentorship program was under taken by the author under the auspices of a senior surgeon at the same hospital he practices in. This was under taken to learn the Laparoscopic Transabdominal Pre - Peritoneal herniorrhaphy technique (TAPP). This involved supervision of the first twenty operative procedures. Once there was satisfaction with the technique the remaining surgical procedures were performed independently without supervision.
\end{abstract}

Over a four-year duration, fifty-nine procedures were performed by the first author. Data was assessed retrospectively with the use of an outcome questionnaire adapted from the EuroQol@ questionnaire.

Results: A patient centered outcomes questionnaire was employed to determine the efficacy of the mentorship program. This was done through a retrospective audit of the first four years of this advanced technique. An established short quality of life questionnaire, the EuroQol@ was used and adapted for laparoscopic hernia surgery 94 percent of patients would recommend the surgeon to other patients for this surgery. There were no hernia recurrences and three patients had inguinodynia, all of whom were managed conservatively

Discussion: The patient centered outcomes clearly demonstrate satisfaction with the surgical mentorship program. While this was a smal study with insufficient follow up for long term post - operative complications, it does show the feasibility of patient centered outcomes in determining the effectiveness of surgical mentorship.

Keywords: Patient centered outcomes; Laparoscopic inguinal herniorrhaphy; Surgical mentorship

\section{Introduction}

Enhanced laparoscopic skills are often difficult to obtain for surgeons in private practice and this difficulty is magnified in rural surgery. Given the paucity of general surgeons in rural practice as well as the large geographic area covered, the ability to enhance one's laparoscopic skills is usually obtained at great financial and personal cost to the surgeon and often involves training at a center, a great distance from the surgeon 's hospital. An alternate way to acquire the requisite skill set is to learn from a senior surgeon who is performing the surgery at the same hospital or health region [1]. A surgical mentorship programmer was undertaken by the author under the auspices of a senior surgeon at the Victoria hospitain Prince Albert, Saskatchewan, Canada. This involved the learning of the laparoscopic Trans Abdominal Pre-Peritoneal Herniorrhaphy (TAPP) technique. A polypropylene mesh was used and inserted pre-peritoneally and covered by the peritoneal flaps that were raised laparoscopically.

The first twenty repairs were done under supervision of the surgical mentor who was present in the operating room (OR) as an observer. Prior to this the junior surgeon scrubbed in as a surgical assistant with the mentor surgeon to observe the procedure. This occurred for half a dozen procedures before the junior surgeon began performing the procedure under the surgial mentor's supervision. Once there was satisfaction with the surgical technique, the remaining procedures were performed independently without the surgical mentor in the OR. The junior surgeon's laparoscopic expertise extended to cholecystectomy and appendectomy prior toembarkingonthis mentorship programmer.

The mentorship process was facilitated by the OR already being setup for a TAPP approach, the technique employed by the surgical mentor. The OR staff had previouslybeen trained in this advanced technique which facilitated an easier transition for the junior surgeon in terms of the operative setup and surgical instrumentation. The surgical procedures were video recorded with informed consent from the patients. The videos were viewed post operatively by the surgical mentorwho reviewed the surgical technique employed.
Austin J Surg - Volume 5 Issue 7 - 2018

ISSN : 2381-9030 | www.austinpublishing group.com

Pillay et al. (c) All rights are reserved
Citation: Pillay $\mathrm{Y}$ and Abeygunawardena K. The Efficacy of Patient Centered Outcomes in Determining the Effectiveness of Surgical Mentorship in Rural Saskatchewan Austin J Surg. 2018. 5(7): 1149. 
Table 1: Breakdown of the unanswered questionnaires.

\begin{tabular}{|l|c|}
\hline Total number of patients & 59 \\
\hline 1. Number of questionnaires answered & $47(80 \%)$ \\
\hline 2. Unanswered questionnaires: & 12 \\
2.1 Deceased patients & $2(3 \%)$ \\
\hline 2.2 Patients who refused to answer & $2(3 \%)$ \\
\hline 2.3 Patients who could not be contacted & $8(14 \%)$ \\
\hline
\end{tabular}

Figure 1: Euroqol questionnaire [3].

\begin{tabular}{|c|c|}
\hline Patient name & \\
\hline \multirow{4}{*}{ Age } & $<25$ years \\
\hline & $40-40$ \\
\hline & $40-65$ \\
\hline & $>65$ \\
\hline \multirow{2}{*}{ Sex } & Male \\
\hline & Female \\
\hline \multirow{4}{*}{ Duration Post Repair } & $1-6$ months \\
\hline & 12-Jun \\
\hline & $1-2$ years \\
\hline & $>2$ years \\
\hline \multirow{4}{*}{ Reason for Surgery } & Groin pain \\
\hline & Difficulty working \\
\hline & $\begin{array}{l}\text { History of incarceration/ } \\
\text { strangulation }\end{array}$ \\
\hline & Other \\
\hline \multirow{5}{*}{$\begin{array}{l}\text { Global outcome (how much did the } \\
\text { operation help) }\end{array}$} & Helped a lot \\
\hline & Helped only a little \\
\hline & Did not help \\
\hline & Poor outcome \\
\hline & Made things worse \\
\hline \multirow{4}{*}{ Satisfaction with treatment } & Very satisfied \\
\hline & Satisfied \\
\hline & Somewhat dissatisfied \\
\hline & Not Satisfied \\
\hline \multirow{4}{*}{ Patient rated complications } & $\begin{array}{c}\text { Sensory disturbance } \\
\text { (loss of sensation) } \\
\text { Groin problem } \\
\text { (swelling or recurrence) }\end{array}$ \\
\hline & Testicular pain \\
\hline & Hematoma \\
\hline & Sexual dysfunction \\
\hline \multirow{4}{*}{ Bothersomeness of complication } & Not at all \\
\hline & Slightly \\
\hline & Moderately troubled \\
\hline & Very bothered \\
\hline Would you recommend this surgeon to & Yes \\
\hline \multirow[t]{2}{*}{ family and friends for hernia surgery } & Maybe \\
\hline & No \\
\hline
\end{tabular}

This helped to eliminate technical errors and reinforce appropriate surgical techniques, which werefurtherstrengthened by adherence to surgical first principles of the TAPP procedure.Historically, in determining the efficacy of previous mentorship programssurgeons have looked at surgical outcome parameters such as operating time, surgical complications and operative recovery time [2]. This usually addresses the surgeon's outlook on surgery and rarely does it take into consideration, patient centered outcomes.

\section{Material and Methods}

Ethics approval was obtained from the Prince Albert Parkland
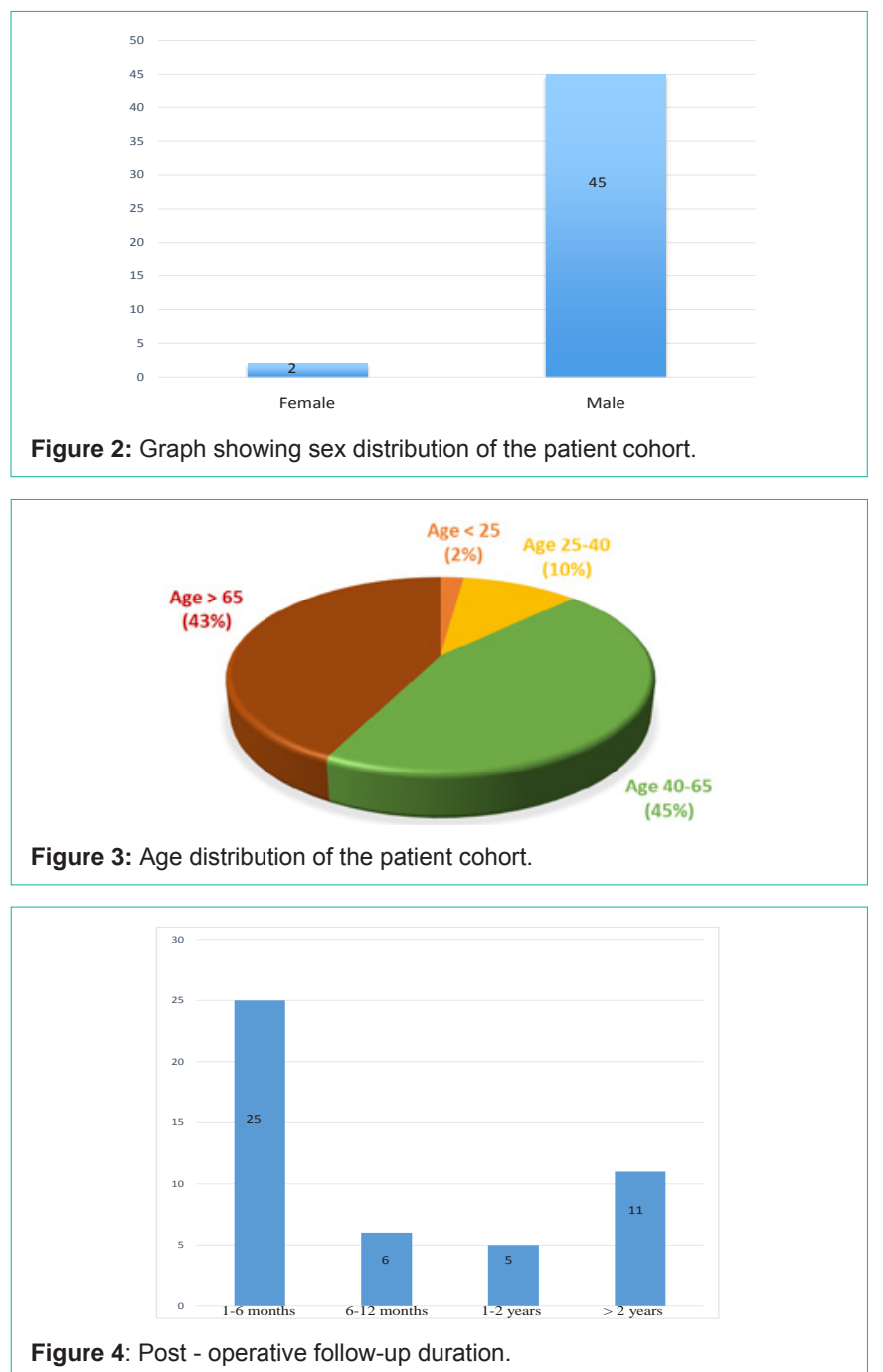

Health Region for this study. This retrospective audit looked at the first 59 laparoscopic inguinal hernia repairs that were performed. This occurred over four years from 2011 to 2015. Emphasis was placed on the duration of four years, hence the odd number of 59 patients. A questionnaire on patient outcomes was used and a telephonic interview conducted. This was performed by the medical office assistant and the juniorsurgeon. Data collection took approximately nine months. This was quite a laborious process given the migrant nature of the population in northern Saskatchewan and the lack of up to date contact information on the patient cohort. Data collection often involved contacting the patient's family or friends and leaving messages for them to contact the surgeon's office. Messages were also left with referring family physicians to ask the patient to contact us when they arrived for a medical examination. Despite exhaustive 


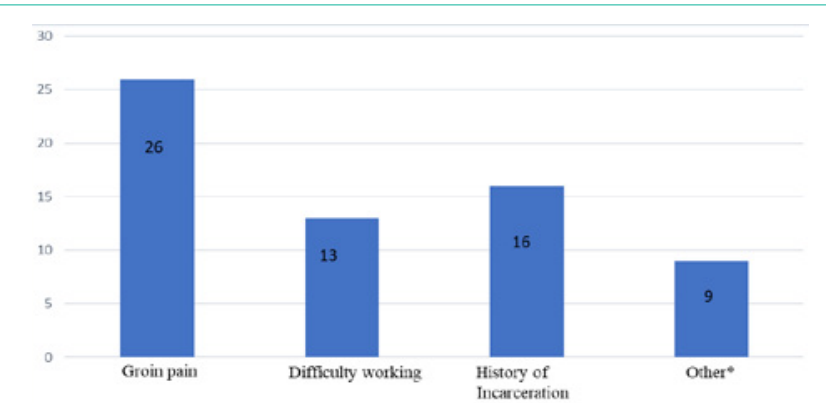

Figure 5: Surgical indications for herniorrhaphy.

\begin{tabular}{|l|l|}
\hline OTHER REASONS & NUMBER OF PATIENTS \\
\hline Physician detected & 2 \\
\hline Popping feeling & 1 \\
\hline Swelling & 1 \\
\hline CT scan detected & 1 \\
\hline Bulge & 2 \\
\hline Tear while walking & 1 \\
\hline Surgical recurrence & 1 \\
\hline
\end{tabular}

Figure 5.1: Other reasons for surgery.

attempts there were eight patients who could not be contacted (Table 1). The surgical questionnaire was adapted from an established short quality of life Questionnaire (Qol), the EuroQol ${ }^{\circ}$ questionnaire [3]. The EuroQol ${ }^{\circ}$ is a standardized instrument for use as a measure of health outcomes. It is applicable to a wide range of health conditions and treatments, and has been validated in many languages (Figures 1-5). This was a standard patient outcome questionnaire adapted for hernia surgery. The adaptation was performed to keep the questions limited to the basic issues of inguinal herniorrhaphy while determining patient demographics as well.

\section{Discussion}

The patient centered outcomes clearly demonstrate that there is satisfaction with this technique (Figures 6-9). The large majority of patients would recommend the surgeon to other patients for surgery and this is demonstrated quite convincingly (Figure 10) with confidence intervals of 83 to $99 \%$. Patient oriented assessments have recently come into vogue as a means of assessing surgical outcomes and the quality of patient care [4]. There has been a paucity of data in laparoscopic inguinal herniorrhaphy to date. There are very few studies that have examined the effect of patient centered outcomes on surgical mentorship programs and this study serves to address that issue as a means of assessing the level of surgical training outside recognized norms. The common hernia-specific questionnaires, the Inguinal Pain Questionnaire (IPQ) ${ }^{\odot}$ and the Carolina Comfort $\mathrm{Scale}^{\odot}$, are not widely used at present. Both instruments are relatively long, with 23 and 18 items respectively, and the IPQ can only be used retrospectively [5]. Whilst these studies are multidimensional and assess factors such as groin pain, functional issues and quality of life, the length of the questionnaire can be a burden for patients to complete and this often affects their willingness to participate in a clinical study [5]. It is for this reason that the questionnaire length was kept to a minimum, as patients were difficult to contact and also had a great variability in their educational background. The core outcomes that were addressed have been shown to have the same effects and

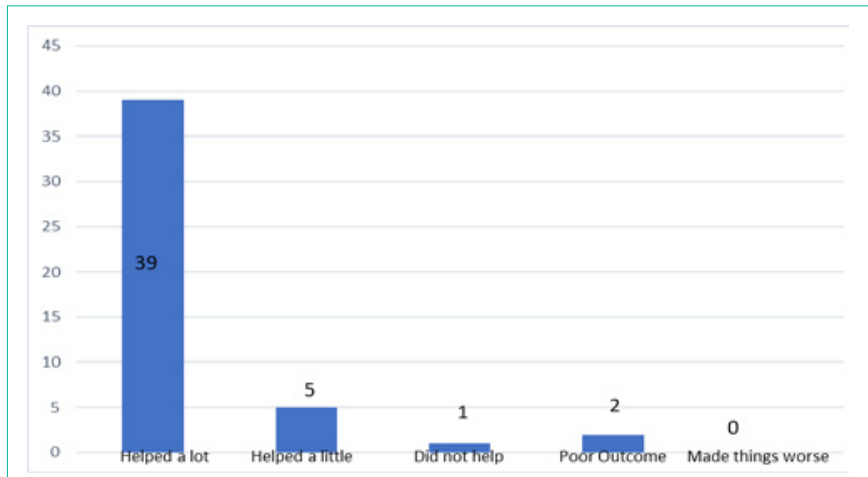

Figure 6: Global outcome of TAPP repair.

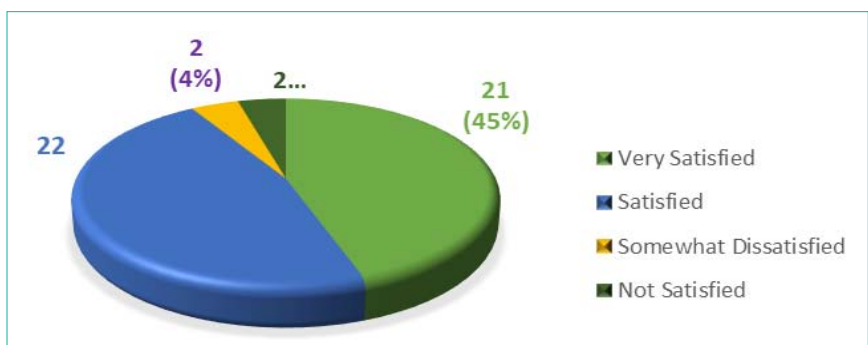

Figure 7: Satisfaction with treatment.

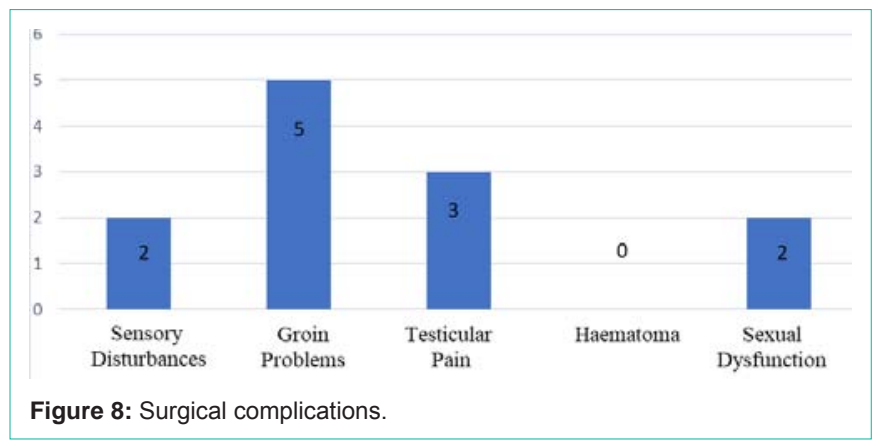

deliver comparable information to the much longer IPQ and Carolina Comfort Scale questionnaires [6,7]. There were no major surgical complications such as bleeding, bowel injury, bladder injury, sepsis or spermatic chord damage. There were no hernia recurrences.

The overwhelming majority of patients were male and elderly. The two female patients, one had a femoral hernia, diagnosed intraoperatively whilst the other had an inguinal hernia. Both made uneventful recoveries. Indications for surgical intervention were quite varied (Figures $5 \& 5.1$ ).

There were two patients who had demised since their surgery and their deaths were unrelated to the hernia surgery as per conversations with their family members.

Two patients refused to participate in the study and would not provide a reason for their lack of participation. The groin problem, which referred to a feeling of fullness over the mesh site, was most probably due to mesh fibrosis. Most of them had settled at the time of the telephonic interview and were present mainly in the first six months after surgery. Two patients complained of sensory disturbances (Figure 8) over their anterior thigh and this fit the 

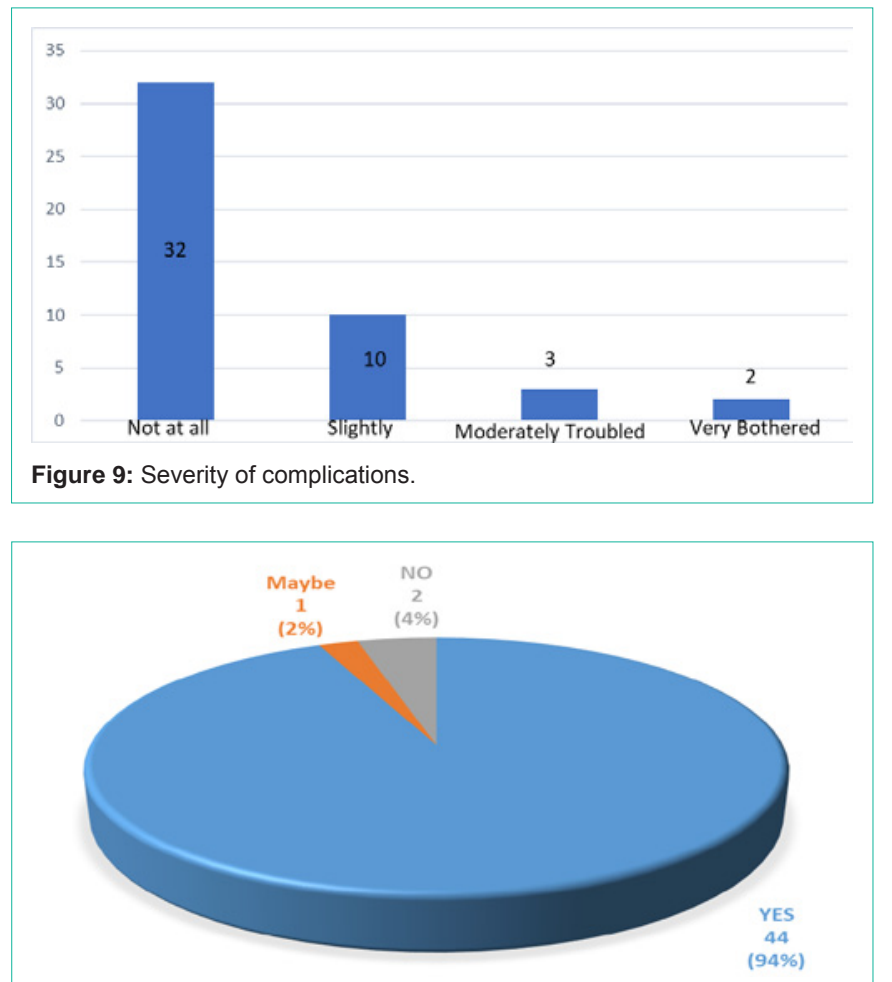

Figure 10: Surgeon recommendation to other patients.

distributive pattern of an injury to the lateral cutaneous nerve of the thigh. One made a complete recovery while the second patient had the area of anesthesia reduced over his thigh but did not make a complete recovery. This patient indicated that it has not impacted his quality of life at all. Inguinodynia was observed in 3 patients, 2 of whom settled with conservative management whilst the third was referred to a pain clinic for follow-up (Figure 8). Interestingly the patient indicated that he would still recommend the surgeon for this surgery to other patients.

His was a recurrent hernia repair following an open repair 40 years ago. The surgery itself was quite difficult with extensive dissection of the groin no doubt due to the extensive fibrosis of the previous repair. The inguinodynia was not a totally unexpected occurrence. His pain control has improved since visiting a pain clinic and it no longer impacts his daily activities. All complaints of inguinodynia were reported with the use of titanium staples and have now tapered off completely since we began using absorbable tacks to attach the mesh. Another adaptation to reduce the inguinodynia incidence was to not dissect out the sensory nerves on the psoas muscle. This includes the genital branch of the genitofemoral nerve and the lateral cutaneous nerve of the thigh in the "area of gloom". It has been postulated that the mesh fibrosis incorporates the exposed nerve in the inflammatory process with the resultant inguinodynia. This has been greatly reduced by not exposing the nerve during surgical dissection.

There were two patients who indicated sexual dysfunction as a complication of their surgery. This was a temporary issue and both had resolution of their sexual dysfunction in the first six months after surgery. There was no sexual dysfunction preoperatively. During surgical follow up they were offered referral to a urologist but both declined the referral electing instead for conservative management. Amongst the patients not satisfied with treatment (Figure 9), one had a poor experience with stopping his XareltoR (Factor Xa inhibitor) and the second patient while having no complications from surgery had a poor hospital experience on the day of surgery. This contributed to his overall feeling of dissatisfaction with the surgical process. His dissatisfaction stemmed from severe nausea and vomiting in the recovery area which he felt was inadequately treated. The first patient had bleeding issues at his wound sites after restarting his Xareltopost operatively. He had a similar issue with a previous surgical procedure prior to his hernia surgery. Both patients made uneventful recoveries. One of the shortcomings of this study is the small number of patients. Given that this is a rural hospital in northern Saskatchewan, Canada it will take time to derive a statistically significant patient cohort.

The follow-up duration is another issue as we only have four years of surgical follow up (Figure 4). We will need at least a decade of follow-up to assess the viability of the surgical mentorship programme based on known long term complication rates of hernia surgery. Given the large geographic area, and the migrant nature of the population this may be quite a challenge to accomplish. The lack of pre-operative baseline values for these patients presents a challenge as well. The change in attributes of the patients from baseline to the early post operative phase is especially important in evaluating the development of chronic complications in the patient cohort $[8,9]$. This will need to be addressed in the future as this allows findings to be easily reproduced and is significantly important in clinical practice outcomes.

The junior surgeon conducting telephonic interviews could have had an adverse effect influencing patient opinion. To mitigate this effect, patients were asked to answer as honestly as possible to help provide a more accurate view of the surgery being undertaken as well as the complication rates. They were informed that this could help reduce the complication rates for future surgeries. What remains crystally evident however is the absolute feasibility of the surgical mentorship programme at the Victoria hospital in northern Saskatchewan. The complication rates were well within the norms of published complication rates from previous studies [10,11]. 20\% of our surgical cohort (12/59) (Figure 8) reported post -operative complications compared to the $25 \%$ reported by Staerkle et al. [11] and Frannerby et al. [10] who reported a $27 \%$ complication rate in the Swedish hernia register. This was in stark contrast to the $5 \%$ complication rate reported by the surgeons themselves in the Swedish hernia registry.

This was reinforced by the findings of the patients involved, which confirmed our original hypothesis. What is required now is a multi centre study involving a large patient cohort and an adequate long term follow up of at least 5 years.

\section{Conclusion}

The complication rates and patient outcomes demonstrate clearly that surgical mentorship for enhanced laparoscopic skills can be accomplished even with limited resources in a rural hospital while at the same time providing a durable alternative to acquiring advanced laparoscopic training. Patient centered outcomes can be a viable alternative in the assessment of surgical training in rural 
Saskatchewan, Canada. Larger comprehensive multicenter studies are now needed to ensure complete validity of our original hypothesis.

\section{References}

1. Pouya E, Lauren EF, Kevin CC. Mentorship in surgical training: a systematic review. HAND. 2012; 7: 30-36.

2. Niitsu H, Hirabayashi N, Yoshimitsu M, Mimura T, Taomoto J, Sugiyama Y, et al. Using the Objective Structured Assessment of Technical Skills (OSATS) global rating scale to evaluate the skills of surgical trainees in the operating room. Surg Today. 2013; 43: 271-275

3. Rabin R, de Charro F. EQ-5D. A measure of health status from the EuroQol Group. Ann Med. 2001; 33: 337-343.

4. Kurzer M, Kark AE, Hussain T. Hernia repair: Outcomes other than recurrence should be analysed. BMJ 2008; 336: 1033

5. Heniford BT, Walters AL, Lincourt AE, Novitsky YW, Hope WW, Kercher KW Comparison of generic versus specific quality-of-life scales for mesh hernia repairs. J Am Coll Surg. 2008; 206: 638-644.
6. Pellis'e F, Vidal X, Hern'andez A, Cedraschi C, Bag'o J, Villanueva C Reliability of retrospective clinical data to evaluate the effectiveness of lumbar fusion in chronic low back pain. Spine. 2005; 30: 365-368.

7. Mannion AF, Elfering A, Staerkle R, Junge A, Grob D, Semmer NK, et al. Outcome assessment in low back pain: how low can you go? Eur Spine J. 2005; 14: 1014-1026.

8. Fr"anneby U, Gunnarsson U, Wollert S, Sandblom G. Discordance between the patient's and surgeon's perception of complications following hernia surgery. Hernia. 2005; 9: 145-149.

9. Heniford BT, Walters AL, Lincourt AE, Novitsky YW, Hope WW, Kercher KW. Comparison of generic versus specific quality-of-life scales for mesh hernia repairs. J Am Coll Surg. 2008; 206: 638-644

10. Fr"anneby U, Sandblom G, Nyren O, Nordin P, Gunnarsson U. Self-reported adverse events after groin hernia repair, a study based on a national register. Value Health. 2008; 11: 927-932.

11. RF Staerkle, P Villiger. Simple questionnaire for assessing core outcomes in inguinal hernia repair. British Journal of Surgery. 2011; 98: 148-155. 